\title{
Julien Barry et les «synapses neurosécrétoires» (1954-1973)
}

\author{
Par Jean Jacques Dreifuss
}

Deux grands systèmes de communication entre cellules assurent le réglage des processus biologiques dans l'organisme. La question de savoir en quoi ces deux ensembles - le système nerveux d'un côté, les glandes endocrines de l'autre - se distinguent, de même que celle de la nature de leurs interactions, constituent un chapitre fascinant de l'histoire des idées.

Les adjectifs neuroendocrine ou neurosécrétoire symbolisent la double nature, nerveuse et hormonale, de certaines cellules dont les prototypes, chez les vertébrés, sont les cellules hypothalamo-hypophysaires. J'ai évoqué à une autre occasion l'évolution de nos conceptions sur les fonctions endocrines du lobe postérieur de l'hypophyse, conceptions qui ont joué un rôle clé dans la genèse de la neuroendocrinologie ${ }^{1}$. Nous voyons dans le présent article qu'à peine cette discipline avait-elle acquis droit de cité que de nouvelles observations auraient nécessité un réajustement conceptuel. Nous analyserons les résistances initiales, mais aussi la rectification tardive des prémices qui fondaient la neuroendocrinologie.

\section{Les observations de 1954}

La notion que les produits de sécrétion qui gagnent la voie sanguine à partir de la posthypophyse servent par ailleurs aussi de médiateurs synaptiques dans le système nerveux central est due au professeur Julien Barry et date de 1954.

Né à Cahors le 6 octobre 1918, Julien Marcel Barry était alors âgé de 36 ans. Il avait étudié à l'Ecole de santé militaire de Lyon, obtenant le doctorat en médecine en 1943. Sous l'égide du professeur Policard ${ }^{2}$ et surtout de $\mathrm{J}$. Broussy ${ }^{3}$ - avec qui il signa plusieurs articles portant notamment sur les cellules intestinales et sur le «lait de pigeon» - il s'était spécialisé en histologie, avait enseigné une année à la Faculté de Médecine de Hanoï, puis réussi le concours d'agrégation en 1952. La même année, il était devenu chercheur libre (en raison de son statut de médecin militaire d'active) dans le laboratoire d'histologie de la Faculté de Médecine de Nancy, que dirigeait le professeur Remy Collin ${ }^{4}$. 
C'est ce dernier qui, s'étant de longue date intéressé à l'histologie de l'hypophyse, orienta Barry vers la neuroendocrinologie. Au début des années 1950, celle-ci était en train de conquérir, après deux décennies d'opposition, ses lettres de noblesse. G. W. Harris ${ }^{5}$ avait publié en 1948 ses premiers travaux sur le contrôle hypothalamique des fonctions de l'hypophyse antérieure, dont les résultats aboutissaient à la notion d'une action de nature humorale. L'imprégnation totale des neurones hypothalamo-posthypophysaires par Wolfgang Bargmann ${ }^{6}$ utilisant la coloration de Gomori ${ }^{7}$ avait enfin corroboré le concept de «glande endocrine diencéphalique» que Ernst Scharrer ${ }^{8}$ avait proposé vingt ans auparavant.

Le 11 février 1954, devant la Société des Sciences de Nancy, Barry rapporte l'existence de terminaisons particulières péri-nerveuses, correspondant à de véritables "synapses neurosécrétoires»; l'élément afférent de ces synapses est formé, écrit-il, de fibres neurosécrétoires qui véhiculent la même substance de Gomori que les fibres de la voie hypothalamo-hypophysaire ${ }^{9}$.

Puis, le 8 avril 1954, devant la même Société, il déclare avoir décelé de nouvelles voies «Gomori-positives» dans le cerveau. Ces voies nerveuses se terminaient dans ce qu'il proposait d'appeler des «synapses neurosécrétoires» et qui sont illustrées dans la figure 1. De telles terminaisons, affirmaitil, affectent, du point de vue morphologique, l'aspect de synapses typiques et possèdent vraisemblablement du point de vue physiologique, la signification de véritables articulations chimiques inter-neuronales fonctionnant par cession de substance. Et il concluait: En supposant même que ces voies, ce qui est peu probable, soient strictement limitées au matériel précis de notre étude, elles suffiraient néanmoins à établir d'une part, que la voie hypothalamo-hypophysaire n'est pas la seule voie neurosécrétoire (...) et, d'autre part, que la substance de Gomori est susceptible d'exercer son action à des niveaux différents du névraxe et souvent très éloignés du complexe hypophysaire ${ }^{10}$.

Avec les moyens techniques à disposition, soit le microscope photonique et la coloration de Gomori, il était difficile d'aller plus loin dans l'analyse. En 1958 et 1961, Barry décrivait la présence dans des extraits de cerveau de la chauve-souris ${ }^{11}$ et du cobaye de substances qui, comme l'ocytocine, contractaient l'utérus. H. et E. Legait ${ }^{12}$ de leur côté détectèrent la présence d'une activité antidiurétique dans des extraits de cerveau du poulet ${ }^{13}$. Mais ces données reposaient sur une méthode d'isolement assez rudimentaire, de sorte que la spécificité de l'action des extraits pouvait être mise en doute.

Barry, à fin 1956, quitta Nancy pour Besançon où il fut d'abord professeur agrégé, puis titulaire. En décembre 1959, il obtint le grade de 
Figure 1 Quelques types de synapses neurosécrétoires observées par Barry chez Rhinolophus ferrum equinum (a,b,d,e) et dans le noyau de la strie semi-circulaire chez la souris (c). Figure 2 de l'article de la note 16.
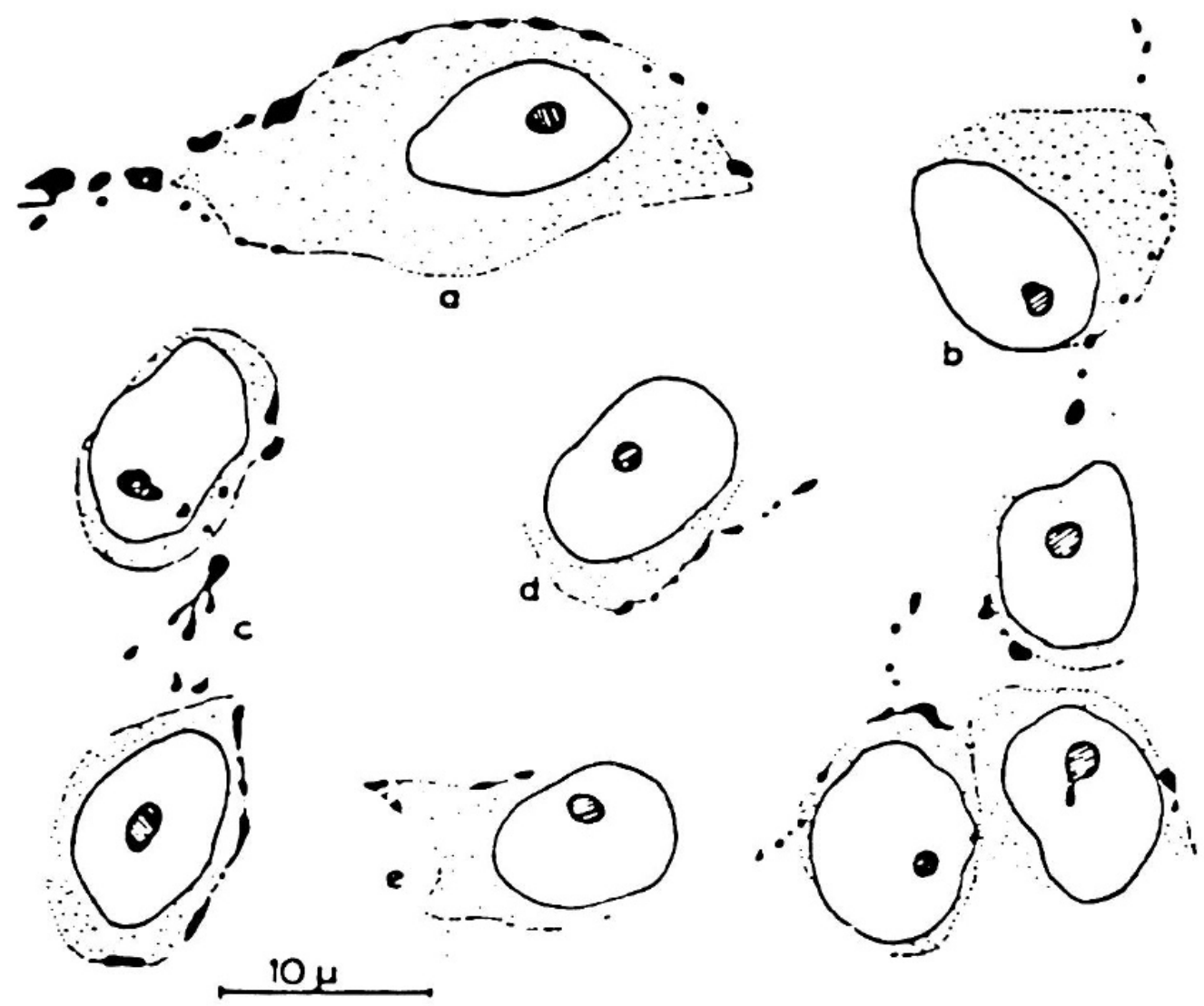

docteur ès sciences avec une thèse dont la partie expérimentale était pour une large part un résumé de ses travaux antérieurs sur les voies neurosécrétoires extra-hypophysaires des mammifères ${ }^{14}$. Les deux années suivantes, Barry enseigna à la Faculté de Médecine et de Pharmacie d'Alger. Après l'accession de l'Algérie à l'indépendance, il fut reclassé en 1962 à Lille, où il occupa jusqu'à sa retraite la chaire d'histologie et d'embryologie à la Faculté de Médecine; il y fut aussi chef du service de cytologie endocrinologique au Centre hospitalier régional. C'est à Lille que nous le retrouverons dans les années soixante-dix, lorsque la notion de «synapses neurosécrétoires» s'imposera enfin à la communauté scientifique.

Pendant près de deux décennies, en effet, l'impact des observations de Barry fut quasi nul, même en France, et cela bien que des données semblables provenant d'autres espèces fussent rapportés par $H$. et E. Legait qui travaillaient également à Nancy. 


\section{Absence d'impact, pourquoi?}

Barry obtint ses premiers et ses meilleurs résultats alors qu'il utilisait un matériel particulier - la chauve-souris en hibernation - de sorte qu'on a pu mettre en doute l'importance d'une observation obtenue dans une espèce donnée et dans une situation particulière. Or cette cause ne devrait pas avoir joué un rôle déterminant, car Barry obtint peu après des images semblables, bien que plus discrètes, chez l'animal non hibernant et dans d'autres espèces de mammifères ${ }^{15}$.

On pourrait penser que l'absence d'impact des observations de Barry était dû à leur publication en langue française et dans des revues scientifiques à faible diffusion. Si elles ont pu de la sorte être occultées, elles n'ont cependant pas pu être ignorées, car Barry a présenté ses observations aux réunions annuelles de l'Association des Anatomistes, à Gênes en 1954 et à Paris en 1955. De plus, il a participé en août 1955 à un congrès qui s'est tenu aux Pays-Bas ${ }^{16}$, où l'occasion lui a été donnée de décrire les «synapses neurosécrétoires» à un public averti: dans la même session où parlait Barry, d'autres communications furent présentées notamment par John C. Eccles, John Z. Young et Frédéric Bremer, tous neurobiologistes réputés. Plus important pour le sujet qui nous concerne, on note parmi les participants à cette réunion de Groningen le nom de Wolfgang Bargmann, ainsi que de celui d'Ernst Scharrer, deux des principaux hérauts de la neuroendocrinologie (Figure 2). Bargmann prit d'ailleurs la parole lors de la discussion qui suivit la présentation de Barry pour signaler qu'il venait de déceler la présence de fibres neurosécrétoires dans un organe extrahypophysaire, la glande pinéale du hérisson.

Le silence qui fut opposé aux conceptions de Barry a donc nécessairement d'autres raisons. Il ne fut pas combattu, mais ignoré; à notre avis, ignoré intentionnellement par des contemporains qui n'avaient que faire de ses «synapses neurosécrétoires». D'ailleurs, il n’a même pas été le premier à déceler l'existence des fibres Gomori-positives en dehors de la voie neurosécrétoire hypothalamo-hypophysaire. L'existence chez certains reptiles d'une voie neurosécrétoire à destination épithalamique avait déjà été décrite en 1951, aussi bien par E.Scharrer ${ }^{17}$ que par W. Hild. Ces auteurs avaient cependant conclu qu'elle se terminait par le biais de seuls contacts neurovasculaires.

Avec l'imprégnation sélective des neurones hypothalamo-posthypophysaires en 1949, la neuroendocrinologie avait trouvé de solides assises. La 


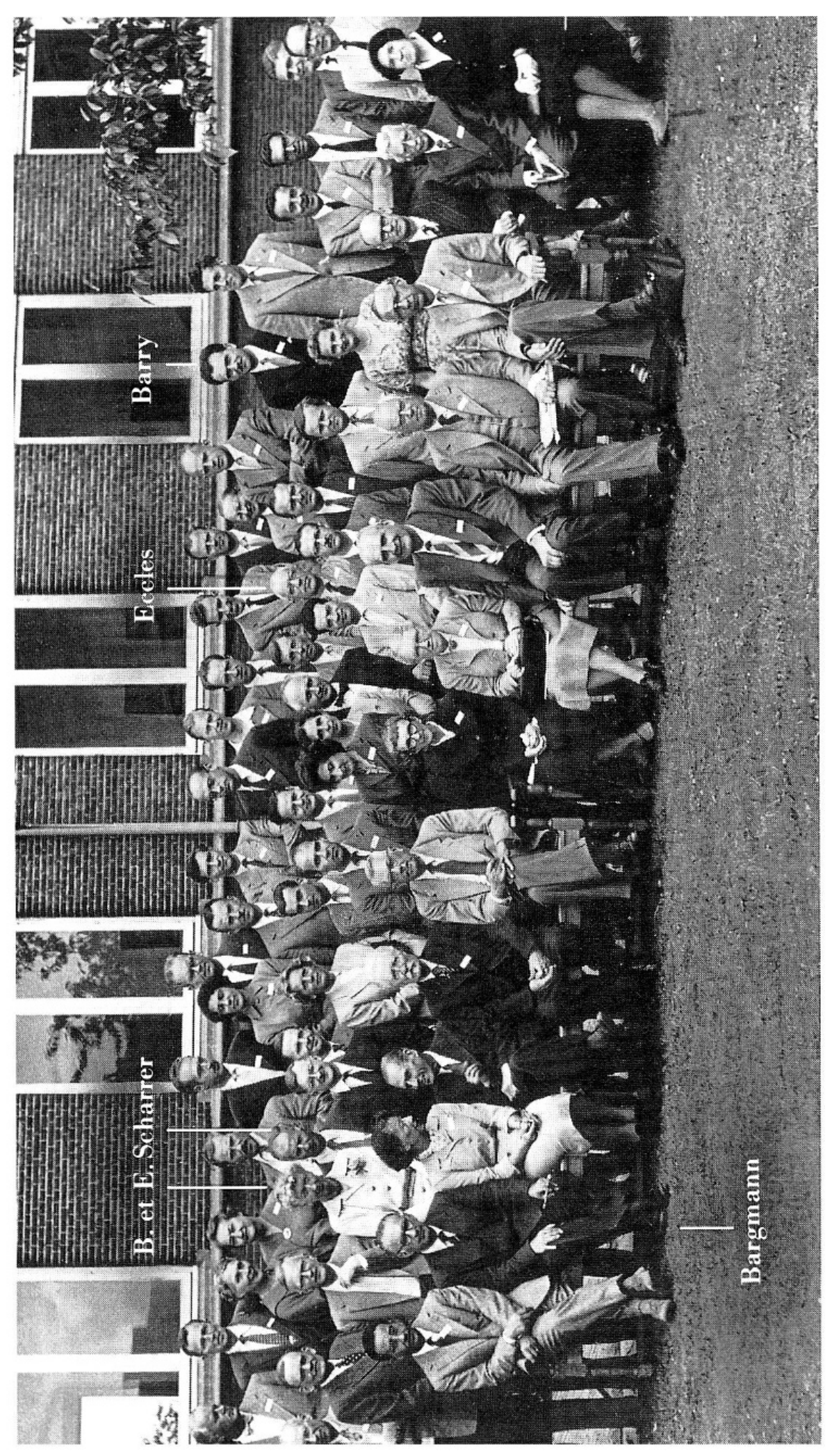

$\stackrel{\infty}{\frac{0}{2}} \frac{\pi}{\pi}$

ป อ

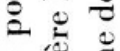

芯恼

$=\frac{T}{a}$

है

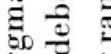

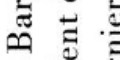

$\geqslant$.

.

员婴

焉

$\Xi \overline{\check{D}}$

$\approx$ \%

颉苛

$\exists \stackrel{\Xi}{9}$

范

b.

कृ

$4 \widetilde{\pi}_{0}$

80 프

$.9 \frac{90}{3}$

ํㅗㅇ

ป

乙.

\%

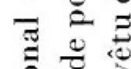

ป气

ॠ

总

g.

ए

总.

g

क

苟寻空

ป

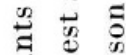

ํำ

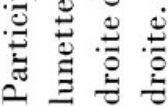

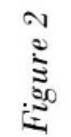


cellule neurosécrétoire est alors conçue comme constituant l'unique lien entre les deux grands systèmes de commande de l'organisme, le système nerveux et le système endocrine. Avec son corps cellulaire et son arborisation dendritique localisés dans le système nerveux et avec ses terminaisons axonales périvasculaires et extracérébrales, elle possède une directionalité: c'est une cellule «nervifugue» et «hémotrope». Ce n'est pas au moment où ce concept venait de s'imposer que ceux qui l'avaient forgé étaient disposés à l'abandonner ou à gommer la distinction qu'ils établissaient entre neurones et cellules neurosécrétoires.

Dans un article de synthèse paru en 1954, l'année des publications originales de Barry, E. et B. Scharrer opposaient comme suit les propriétés des cellules neurosécrétoires de celles des neurones: die neurosekretorischen Zellen dagegen produzieren Hormone, die nicht für die nervöse Funktion der Neuronen nötig sind, sondern für physiologische Abläufe außerhalb des nervösen Geschehens ${ }^{18}$. Par la suite, certains mirent en doute cette distinction, notamment après que la microscopie électronique eut révélé - dans le cytoplasme des cellules neurosécrétoires comme dans celui de neurones - la présence des mêmes grains contenant du produit de sécrétion. E. Scharrer avait rétorqué en 1961: The definition of the neurosecretory cell is not solely based on the presence of neurosecretory granules but also on the fact that neurosecretory cells do not form synapses with other neurones or effector organs $^{19}$. Mais cette définition dut, elle aussi, être révisée. Il s'avéra en effet que les cellules neurosécrétoires peuvent établir des contacts de type synaptique avec des cellules dans des organes effecteurs, notamment dans le lobe intermédiaire de l'hypophyse.

Les implications théoriques de ses observations et ce que celles-ci pouvaient avoir d'hérétique pour une neuroendocrinologie entièrement tournée vers l'hypophyse n'avaient pas échappé â Barry. Aussi avait-il remarqué dès 1954 que les synapses neurosécrétoires représentaient non seulement une notion nouvelle mais la réalisation d'une éventualité qui avait été explicitement écartée sur la base des recherches antérieures ${ }^{20}$. Et dans sa thèse de doctorat parue sept ans plus tard Barry réitérait son opinion selon laquelle les voies extra-hypophysaires, ainsi que nous l'avons suggéré dès 1954, constituent la démonstration histologique d'une action nerveuse centrale des hormones posthypophysaires; elles représentent probablement des voies par lesquelles l'hypothalamus tient sous sa dépendance (ou peut influencer) certains territoires sus- ou sous-jacents, vraisemblablement affectés à des régulations végétatives $^{14}$. 


\section{"Neurosecretory synapses"}

Sir Francis Knowles, inaugurant en 1973 le $6^{\mathrm{e}}$ Symposium International sur la Neurosécrétion, définissait une fois encore la cellule neurosécrétoire à la manière de Scharrer, toutefois sans inclure l'absence de contacts directs avec des organes effecteurs: It receives synaptic efferents but itself makes no efferent synapses with other neurones ${ }^{21}$. En fait et contrairement aux vues de Knowles, ce symposium réunissait plusieurs de ceux grâce à qui la notion de «synapses neurosécrétoires» fut adoptée, sous un autre terme il est vrai, que Bargmann avait proposé en 1967, celui de synapse peptidergique 22 .

La situation qui prévalait en neuroendocrinologie en 1973 contrastait fortement avec celle qui avait eu cours deux décennies plus tôt. Des données provenant d'horizons divers avaient progressivement ébranlé les notions qui avaient servi à distinguer les cellules neurosécrétoires des neurones. Allusion a déjà été faite à quelques apports dus au microscope électronique. Par ailleurs, les cellules neurosécrétoires s'étaient avérées capables de produire des potentiels d'action et ceux-ci, comme dans un «banal» neurone, déclenchaient la sécrétion. De plus, la liste des neurones qui produisent et sécrètent des peptides s'était fortement allongée. Un travail biochimique de grande envergure avait permis à Roger Guillemin, Andrew Schally et à leurs collaborateurs respectifs de découvrir de nouvelles neurohormones d'origine hypothalamique. Un tripeptide ayant une action sur les cellules thyréotropes de l'hypophyse antérieure fut isolé en 1969. Puis en 1971, fut annoncée la caractérisation et la synthèse d'un décapeptide à action gonadotrophique, le luteinizing hormone releasing factor (LRF) ou luteinizing hormone releasing hormone (LHRH), selon une autre terminologie. Ces découvertes, rendues possibles par une technologie chimique s'effectuant à une échelle qui n'avait pas eu de précédent et facilitées par un dosage immunologique dont la rapidité dépassait largement celui du bioétalonnage traditionnel, eurent un immense et immédiat écho. Elles valurent en 1977 à R. Guillemin, A. Schally et à Roselyn Yalow - à qui on devait le principe de l'immunoétalonnage - le prix Nobel de médecine et de physiologie ${ }^{23}$.

Julien Barry s'intéressait de longue date aux mécanismes hypothalamiques qui règlent les fonctions de reproduction. Aussi l'isolement du LRF ne pouvait-il le laisser indifférent. Avec un immunsérum qui contenait des anticorps dirigés contre le LRF de synthèse qu'il obtint de Maurice P. Dubois ${ }^{24}$, Barry et ses collaborateurs réussirent à localiser par immunofluorescence les neurones à LRF présents dans le cerveau du cobaye. Deux brèves 
communications furent présentées en mars et en juin 1973 à l'Académie des Sciences de Paris; à fin septembre, un article complet et abondamment illustré, rédigé en langue anglaise, fut envoyé à une revue scientifique de grande diffusion. Barry y décrivait la localisation des corps cellulaires et le trajet des voies nerveuses contenant le LRF: la plupart des axones se dirigeaient vers l'éminence médiane et s'y terminaient sous la forme des contacts neurovasculaires typiques; d'autres axones, en petit nombre, gagnaient diverses régions du cerveau où ils formaient des «synapses neurosécrétoires à $L R F{ }^{25}$, rappelant à maints égards les «synapses neurosécrétoires à matériel Gomori-positif» de 1954.

Barry était de la sorte dans la situation privilégiée et rare de trouver, à deux décennies d'intervalle, un nouvel argument soutenant la notion qu'un même produit de sécrétion peut servir tantôt de neurohormone-lorsqu'il est déversé dans le flot sanguin - et tantôt de médiateur synaptique - lorsqu'il est sécrété par des axones à parcours intracérébral.

Deux articles parurent la même année dans la revue Science, dans lesquels il était montré que l'injection de LRF à des rates femelles déclenchait après une courte période de latence une posture de réceptivité à l'acte sexuel, une «lordose» ${ }^{26}$. L'auteur de l'un de ces articles, pour expliquer ce comportement, postula that some LRF-producing neurones project, either directly or via axon collaterals, to other neurones concerned with lordosis, as well as to the median eminence. Ce sont précisément ces projections extrahypophysaires qu'observait Barry à cette même époque sous le microscope.

Le Symposium International de 1973 constitua le premier rassemblement de neuroendocrinologistes où le concept de synapse peptidergique eut sa place. J. Barry présenta ses récents résultats sur les neurones à $L_{R F}{ }^{27}$, tandis que G.Sterba y montra des images de «synapses neurosécrétoires» prises au microscope électronique ${ }^{28}$. B. Cross de son côté décrivit des expériences récentes provenant de son groupe qui suggéraient que l'ocytocine - un produit de sécrétion provenant de neurones hypothalamo-hypophysaires «Gomori-positifs» - exerçait un effet direct sur des neurones intracérébraux, effet qui était décelable par l'enregistrement électrophysiologique ${ }^{29}$. Près de vingt ans s'étaient écoulés depuis que Barry, se basant sur des données obtenues avec une coloration cytochimique et le seul microscope photonique, avait prédit que les hormones posthypophysaires devaient exercer une action nerveuse centrale ${ }^{30}$. 
1 Dreifuss J.J. (1981), W.L.Gaines, précurseur du concept de réflexe neuroendocrine, Gesnerus 38: 331-338.

2 Médecin général, titulaire de la chaire d'histologie à Lyon, auteur d'un manuel d'histologie classique.

3 Agrégé à Lyon, puis professeur titulaire à Toulouse. Ses principaux travaux concernent le jabot de pigeon et l'éphithelium intestinal, notamment ses cellules glandulaires.

4 Elève, puis successeur de Pol Bouin à la chaire d'histologie de Nancy (qu'il occupa jusqu'en 1957), le professeur Collin s'était spécialisé en histologie nerveuse et hypophysaire. Il installa avant même la seconde guerre mondiale, une section de Neuroendocrinologie dans son Institut.

5 Geoffrey W. Harris (1913-1971), professeur de physiologie à Londres puis d'anatomie à Oxford; pionnier éminent de la neuroendocrinologie, il a le premier suggéré que le contrôle nerveux des fonctions adénohypophysaires s'effectuait par voie neurovasculaire et via les vaisseaux sanguins du système-porte hypophysaire. Voir Harris G. W. (1948), Neural control of the pituitary gland, Physiological Reviews, 28: 139-179, et l'ouvrage paru sous le même titre en $1955 \mathrm{chez}$ Edward Arnold à Londres. Une bonne biographie scientifique de Harris est due à Marthe Vogt, in Biographical Memoirs of Fellows of the Royal Society, Volume 18, November 1972, p. 309-329.

6 Wolfgang Bargmann (1904-1978), professeur d'anatomie dans diverses universités allemandes, notamment à Kiel. Utilisant la coloration de Gomori (voir note 7), il a montré que l'axone issu de neurones hypothalamiques magnocellulaires innerve le lobe postérieur de l'hypophyse. Voir Bargmann W. (1949), Uber die neurosekretorische Verknüpfung von Hypothalamus und Neurohypophyse, Zeitschrift für Zellforschung und mikroskopische Anatomie, 34: 610-634.

7 Coloration à l'hématoxyline chromique-phloxine utilisée initialement pour différencier les cellules endocrines du pancréas. Voir Gomori G.(1941), Observations with differential stain on human islets of Langerhans, American Journal of Pathology, 17: 395-406. En raison d'une affinité pour les groupements sulfurés, cette coloration révèle aussi les substances sécrétoires présentes dans les cellules hypothalamo-posthypophysaires: vasopressine, ocytocine et neurophysines.

8 Ernst Scharrer (1905-1965), neurobiologiste en Allemagne, puis professeur aux EtatsUnis à partir de 1937. Auteur dès 1928 de nombreux travaux histologiques sur la neurosécrétion hypothalamique dans diverses classes de vertébrés. Pour une synthèse de ses travaux et de ceux de sa femme, voir note 18.

9 Barry J.(1954), A propos de la notion de «voie neurosécrétoire», Bulletin de la Société des Sciences de Nancy, 13: 49-58.

10 Barry J.(1954), De l'existence de voies neurosécrétoires hypothalamo-télencéphaliques chez la chauve-souris (Rhinolophus ferrum equinum) en état d'hibernation, Bulletin de la Société des Sciences de Nancy, 13: 126-136.

11 Barry J., Besson S.et Lamarche M.(1958), Activité ocytocique des extraits de noyau amygdalien de Rhinolophus ferrum equinum, Annales d'Endocrinologie, 19: 1045-1051.

12 Après avoir enseigné à Fribourg (Suisse), Etienne Legait fut l'agrégé, puis le successeur de Remy Collin à Nancy. 
13 Legait H. et Legait E.(1958), Présence d'une voie neurosécrétoire hypothalamo-habénulaire et mise en évidence d'une activité antidiurétique au niveau des ganglions de l'habenula chez la Poule, Comptes rendus des Séances de la Société de Biologie, 152: 828-830.

14 Barry J.(1961), Recherches morphologiques et expérimentales sur la glande diencéphalique de l'appareil hypothalamo-hypophysaire, Annales scientifiques de l'Université de Besançon. $2^{\mathrm{e}}$ série. Zoologie et Physiologie, Fasc. 15, 135 p.

15 Barry J. (1954), Neurocrinie de substance neurosécrétoire d'origine hypothalamique, Comptes rendus des Séances de la Société de Biologie, 148: 1459-1460. Il y écrivit: Cette neurocrinie de substance neurosécrétoire que nous avons observée de façon indiscutable chez la Chauve-Souris n'est certainement pas limitée aux Cheiroptères. Chez la Souris blanche, par exemple, quoique de façon plus discrète, nous avons qu l'observer au niveau du corps strié.

16 Barry J. (1956), De l'existence probable de synapses interneuronales de type neurosécrétoire, in Progress in Neurobiology, Proceedings of the First International Meeting of Neurobiologists (Groningen, 1955), edited by J. Ariëns Kappers, Amsterdam, Elsevier, p. 36-44.

17 Scharrer, E. (1951), Neurosecretion. X.A relationship between the paraphysis and the paraventricular nucleus in the garter snake (Thamnophis sp.), Biological Bulletin, 101: 106-113.

18 Scharrer E. and Scharrer B. (1954), Neurosecretion, Handbuch der mikroskopischen Anatomie des Menschen, 6. Band, 5. Teil, Berlin, Springer, p. 953-1066.

19 Cité par F. Knowles, voir note 21.

20 Barry J.(1954), Neurocrinie et synapses «neurosécrétoires», Archives d'Anatomie microscopique et de Morphologie expérimentale, 43: 310-320.

21 Knowles F. (1974), Twenty Years of Neurosecretion, in: Neurosecretion-The Final Neuroendocrine Pathway (VI International Symposium on Neurosecretion, London 1973), edited by F. Knowles and L. Vollrath, Berlin, Springer, p. 3-11.

22 Bargmann W., Lindner E. and Andres K. K. (1967), Über Synapsen an endokrinen Epithelzellen und die Definition sekretorischer Neurone. Untersuchungen am Zwischenlappen der Katzenhypophyse, Zeitschrift für Zellforschung, 77: 282-298. On y lit: It is proposed that in analogy to cholinergic, adrenergic and aminergic neurones, those cells which synthesize octapeptide hormones should be termed peptidergic neurones. It is further suggested to speak of peptidergic synapses if the terminals of such neurones are establishing contact with cells of an endocrine organ.

23 Les discours pronocés par les lauréats à Stockholm lors de la remise du Prix donnent un bon aperçu de leurs travaux respectifs.Voir Yalow R.S. (1978), Radioimmunoassay: A probe for the Fine Structure of Biological Systems, Science, 200: 1236-1245; Schally A. V. (1978), Aspects of Hypothalamic Regulation of the Pituitary Gland. Its Implications for the Control of Reproductive Processes, Science, 202: 18-24; Guillemin R. (1978), Peptides in the Brain: The New Endocrinology of the Neuron, Science, 202: 390-402.

24 Vétérinaire, directeur de recherche à l'Institut National de la Recherche agronomique à Nouzilly près de Tours, M.P.Dubois est l'auteur d'importants travaux de caractérisation, par immunofluorescence, des cellules productrices des hormones de l'hypophyse antérieure et de l'hypothalamus.

25 Barry J., Dubois, M.P. and Poulain, P. (1973), LRF producing cells of the mammalian hypothalamus. A fluorescent antibody study, Zeitschrift für Zellforschung, 146: 351-366. On y lit: Other axons (or axon collaterals) may be found in various areas (suprachiasmal 
crista, epithalamus, amygdala, mesencephalon) and form circuits recalling the "extrahypophyseal pathways" described for the magnocellular Gomori-positive neurons. These axons are probably concerned in intersegmental regulations involving "neurosecretory synapses", particularly of the axosomatic type.

26 Moss, R.L. and Mc Cann, S. M. (1973), Induction of Mating Behavior in Rats by Luteinizing Hormone-Releasing Factor, Science, 181: 177-179; Pfaff, D. W. (1973), Luteinizing Hormone Releasing Factor Protentiates Lordosis Behavior in Hypophysectomized Ovarectomized Female Rats, Science, 182: 1148-1149.

27 Knowles F. and Vollrath L., Editors (1974), Neurosecretion-The Final Neuroendocrine Pathway (VI International Symposium on Neurosecretion, London 1973). Springer, Berlin, p. 148-153 (notamment Fig. 1).

28 Ibid. p. 38-47 (notamment Fig. 3). Voir aussi Sterba G. (1974), Das oxytocinerge neurosekretorische System der Wirbeltiere, Beitrag zu einem erweiterten Konzept, Zoologisches Jahrbuch, Abteilung für allgemeine Zoologie und Physiologie der Tiere, 78: 409-423.

29 Ibid., p. 115-128 (notamment p. 124).

30 Le présent article est une version remaniée et complétée d'un exposé fait en septembre 1984 à Besançon lors d'une table-ronde sur les communications peptidergiques dans le système nerveux central, organisée par la Société de Neuroendocrinologie. Voir Dreifuss J.J. et Raggenbass M.(1985), Les «synapses neurosécrétoires», hier et aujourd'hui, Annales d'Endocrinologie, 46: 47-53.

\section{Summary}

The concept of "neurosecretory synapses" is due to J. Barry, who in 1954 reported on the existence of "Gomori-positive" synapses in the central nervous system of bats and mice. For nearly two decades this discovery had virtually no impact on the scientific community, since the neuroendocrinologists adhered to the definition of the neurosecretory cell given by Bargmann and Scharrer which implied its exclusive neurovascular and therefore nonsynaptic termination. Under the alternative expression of "peptidergic synapses", the concept of "neurosecretory synapses" became generally accepted after 1973, again thanks in part to J.Barry, who that year discovered a new class of such synapses containing LRF, an hypothalamic neurohormone.

Prof. Jean Jacques Dreifuss

Département de Physiologie

Centre Médical Universitaire

9, avenue de Champel

CH-1211 Genève 4 